\title{
Prenatal Exome Sequencing in Recurrent Fetal Structural Anomalies: Systematic Review and Meta-Analysis
}

\author{
Montse Pauta ${ }^{1}\left(\mathbb{D}\right.$, Raigam Jafet Martinez-Portilla ${ }^{1,2}(\mathbb{D})$ and Antoni Borrell ${ }^{1,3,4, *(D)}$ \\ 1 BCNatal, Institut d'Investigacions Biomèdiques August Pi i Sunyer (IDIBAPS), \\ 08036 Barcelona, Catalonia, Spain; mpauta@clinic.cat (M.P.); raifet@hotmail.com (R.J.M.-P.) \\ 2 Clinical Research Division, Evidence-Based Medicine Department, National Institute of Perinatology, \\ Mexico City 01120, Mexico \\ 3 BCNatal, Department of Obstetrics and Gynecology, Hospital Clínic de Barcelona, \\ 08028 Barcelona, Catalonia, Spain \\ 4 Medical School, University of Barcelona, 08036 Barcelona, Catalonia, Spain \\ * Correspondence: ABORRELL@clinic.cat; Tel.: +34-93-227-9946; Fax: +34-93-227-5605
}

check for updates

Citation:

Pauta, M.; Martinez-Portilla, R.J.;

Borrell, A. Prenatal Exome

Sequencing in Recurrent Fetal

Structural Anomalies: Systematic Review and Meta-Analysis. J. Clin. Med. 2021, 10, 4739. https:// doi.org/10.3390/jcm10204739

Academic Editors: Mar Bennasar, Olga Gómez and Erich Cosmi

Received: 12 August 2021

Accepted: 13 October 2021

Published: 15 October 2021

Publisher's Note: MDPI stays neutral with regard to jurisdictional claims in published maps and institutional affiliations.

Copyright: (c) 2021 by the authors. Licensee MDPI, Basel, Switzerland. This article is an open access article distributed under the terms and conditions of the Creative Commons Attribution (CC BY) license (https:/ / creativecommons.org/licenses/by/ $4.0 /)$.

\begin{abstract}
To determine the diagnostic yield of exome sequencing (ES), a microarray analysis was carried out of fetuses with recurrent fetal structural anomalies (with similar anomalies in consecutive pregnancies). This is a systematic review conducted in accordance with Preferred Reporting Items for Systematic Reviews and Meta-Analyses (PRISMA) criteria. The selected studies describing ES in fetuses with recurrent fetal malformation were assessed using the Standards for Reporting of Diagnostic Accuracy Studies (STARD) criteria for risk of bias. Incidence was used as the pooled effect size by single-proportion analysis using random-effects modeling (weighted by inverse of variance). We identified nine studies on ES diagnostic yield that included 140 fetuses with recurrent structural anomalies. A pathogenic or likely pathogenic variant was found in 57 fetuses, resulting in a $40 \%$ (95\%CI: $26 \%$ to $54 \%$ ) incremental performance pool of ES. As expected, the vast majority ( $86 \%$ : $36 / 42$ ) of the newly identified diseases had a recessive inheritance pattern, and among these, $42 \%$ $(15 / 36)$ of variants were found in homozygosity. Meckel syndrome was the monogenic disease most frequently found, although the genes involved were diverse. The ES diagnostic yield in pregnancies with recurrent fetal structural anomalies was 40\% (57/140). Homozygous disease-causing variants were found in $36 \%(15 / 57)$ of the newly identified monogenic disorders.
\end{abstract}

Keywords: exome sequencing; diagnostic yield; prenatal diagnosis; fetal structural anomalies; recurrent anomalies

\section{Introduction}

Structural fetal anomalies occur in approximately $2.5 \%$ of pregnancies [1], and at present most of them can be identified by ultrasound examination during pregnancy. Classically, when a fetal structural anomaly was detected prenatally, fetal karyotyping was able to reveal a chromosomal anomaly in $14 \%$ of the cases [2]. Currently, a chromosomal microarray analysis is preferred because it provides up to a $6-10 \%$ incremental diagnostic yield above the fetal karyotype [3,4]. Sometimes, in a subsequent pregnancy a similar congenital anomaly can be observed. The frequency of recurrent fetal anomalies in singleton pregnancies of the general population has been reported to be $0.03 \%(301 / 872,493)$ by the Northern England Congenital Abnormality Survey [5].

Exome sequencing (ES) enables the assessment of the coding regions of more than 20,000 genes, which comprise approximately 1 to $2 \%$ of the genome. Nowadays, ES is increasingly being used prenatally in fetuses with structural anomalies and normal microarrays. To simplify interpretation and minimize inconclusive findings, the analysis can be restricted to the coding sequences of the OMIM (Online Mendelian Inheritance in Man) genes (clinical or medical ES), or to those genes previously described to be associated 
with a specific condition (gene panels), instead of interpreting the whole exome (whole ES). Studies using whole-genome sequencing (GS) are still scarce in the prenatal diagnosis field.

It has been shown that, for women whose first pregnancy was affected by a fetal structural anomaly, the absolute recurrence risk of a similar anomaly in the second pregnancy is $2 \%$, resulting in a $4.08 \%$ overall risk of congenital anomalies when an extra $2 \%$ risk for dissimilar anomalies is added. Regarding the recurrence risk (RR) for a similar anomaly, it was considerably elevated $(R R=24)$ [5]. This recurrence risk increases dramatically when an autosomal recessive or an X-linked disease is demonstrated by molecular analysis.

In this study, we aim to perform a systematic review of the literature and meta-analysis to assess the diagnostic yield of ES in fetuses with recurrent structural anomalies and a negative result at microarray or karyotyping.

\section{Methods}

\subsection{Protocol and Registration}

The protocol of this systematic review and meta-analysis on prenatal ES and GS in recurrent fetal structural anomalies was prospectively registered and published in an international open-access database for prospective protocols and can be accessed in the following link (http:/ / doi.org/10.17605/OSF.IO/PDBCZ accessed on 23 April 2021). There is no need for institutional approval in our hospital for systematic reviews and meta-analysis. This meta-analysis adhered to the Preferred Reporting Items for Systematic Reviews and Meta-Analyses (PRISMA) guidelines for randomized controlled trials. The study protocol was agreed to among the authors before running the analysis, and one of them (R.M.), being external to the group, acted as a reviewer.

\subsection{Eligibility Criteria}

Criteria for inclusion in this systematic review were observational studies on pregnancies with the following inclusion criteria: (a) fetuses presenting a recurrent fetal structural anomaly (similar anomalies in consecutive pregnancies); (b) absence of a known familial mutation; (c) negative microarray result, treated as the reference standard; and (d) series with more than two cases in the English language. Positive variants classified as IV or V (likely pathogenic or pathogenic) were determined to be causative of the fetal phenotype. Therefore, variants of uncertain significance (VUS) and secondary findings were not extracted. Either ES or GS applied as a solo (the fetus alone) or trio (fetus and both parents) approach were included. The following studies were excluded: (a) case reports; (b) opinion articles or letters; (c) application of gene panels; (d) studies dealing with specific syndromes or malformations; and (e) when data could not be extracted, and the corresponding author did not provide additional information.

\subsection{Information Sources and Search}

A systematic search was conducted using PubMed, SCOPUS, the Web of Knowledge, and the Cochrane database to identify relevant manuscripts published without time limits. References of relevant publications were manually searched for any additional potentially relevant studies that had been published. The first search was run on 15 November 2020, although an update was extended until 20 March 2021. The following MeSH terms (The Medical Subject Headings) with word variation of "genome" and "exome sequencing" and "fetuses" were used in an attempt to capture as many relevant studies as possible. Terms for "exome sequencing" include "genome", "exome", "exome sequencing", or "whole-exome", and alternative terms for "fetuses" included "fetal", "prenatal diagnosis", and "recurrent fetal malformation".

\subsection{Study Selection}

Abstracts identified as relevant were assessed by two independent evaluators (M.P. and A.B.). If studies complied with the inclusion criteria, full-text articles were reviewed. 
In cases of relevant studies with missing information, the corresponding authors were contacted by e-mail. The search strategy and query syntaxes are depicted in Supplementary S1.

\subsection{Data Collection Process and Data Items}

The following data were extracted onto a datasheet based on a Cochrane Consumers and Communication Review Group data extraction template: countries where the study was carried out, study period, study inclusion criteria, sample size, number of cases with recurrent fetal malformation, sequencing approach, Sanger validation, and results of ES/GS. In the case of a positive result, gene, type of variant, classification of variant, and syndrome or disease caused were extracted.

\subsection{Outcome Measures}

The primary outcome of the study was the diagnostic yield with ES/GS among fetuses with recurrent fetal structural anomalies, unknown Mendelian inheritance, and normal microarray. Diagnostic yield was defined as the number of positive ES/GS results among fetuses with recurrent fetal anomalies over the total number of cases with recurrent fetal anomalies (positive plus negative cases).

\subsection{Assessment of Risk of Bias}

Quality assessment was performed using modified Standards for Reporting of Diagnostic Accuracy (STARD) criteria [6]. The quality criteria deemed most important to optimize accuracy were (a) eligibility criteria described (consecutive or not) and the description or ES/GS approach; (b) specific phenotype study of the recurrent fetal structural anomaly; (c) in depth description of the fetal structural anomaly by ultrasound, postmortem studies, or neonatal examination; and (d) American College of Medical Genetics and Genomics (ACMG) classification used. Each parameter was graded as high, unclear, or low risk of bias. The risk of bias was measured individually by two reviewers (M.P. and A.B.).

\subsection{Strategy for Data Synthesis and Statistical Analysis}

The results extracted were pooled in a meta-analysis. For the primary outcome, incidence was used as the pooled effect size by single-proportion analysis [7] using randomeffects modeling (weighted by inverse of variance), along with the Clopper-Pearson exact method for calculation of confidence intervals [8]. We used single proportion analysis to show the proportion of positive ES/GS among fetuses with recurrent fetal anomalies over the total number of fetuses with recurrent fetal anomalies. This analysis requires only cases among the total population to assess the proportion, which is interpreted as the diagnostic yield. Between-study heterogeneity/variability was assessed using the Tau ${ }^{2}, \mathrm{X}^{2}$ (Cochrane $\mathrm{Q}$ ), and $\mathrm{I}^{2}$ statistics. Results were assessed using forest plots and presented as proportions. Publication bias was visually assessed by funnel plots $[9,10]$, quantified by the Egger method (weighted linear regression of the treatment effect on its standard error) [11], and adjusted using the Copas model for selection bias [12-14]. Statistical analyses were conducted using R studio v1.0.136 (The R Foundation for Statistical Computing; (Boston MA, "meta" package for meta-analysis) package "meta v4.2") [15].

\section{Results}

\subsection{Study Selection and Study Characteristics}

For the scoping review selection progress, 106 studies were initially selected from PubMed focusing on ES/GS and fetuses with a recurrent malformation. After full review, one study was deemed eligible and was included in this review [16]. Studies dealing with specific syndromes or malformations were excluded $[17,18]$. During the interactive review, we found series of structurally abnormal fetuses that included cases with a recurrent anomaly, and only those with more than two cases were considered (Figure 1). After full-text reviewing 29 articles, eight ES studies including fetuses with a recurrent structural 
anomaly were included [19-25]. Two articles with less than two cases of recurrent malformations [26,27], and one in which data extraction was not feasible [27], were excluded. No series on GS were found. Finally, data obtained from our own center and previously presented at international conferences were added [28].

A single study was focused on recurrent anomalies [16], while the remaining eight also included anomalous fetuses with non-recurrent anomalies (the latter were not included in the study). All studies were of high quality according to modified Standards for Reporting of Di-agnostic Accuracy (STARD) criteria (Figure 2). These studies were performed in centers from seven different countries (three from USA and one from each of the following countries: China, Greece, Switzerland, Netherlands, Israel, and Catalonia, Spain (Table 1)).

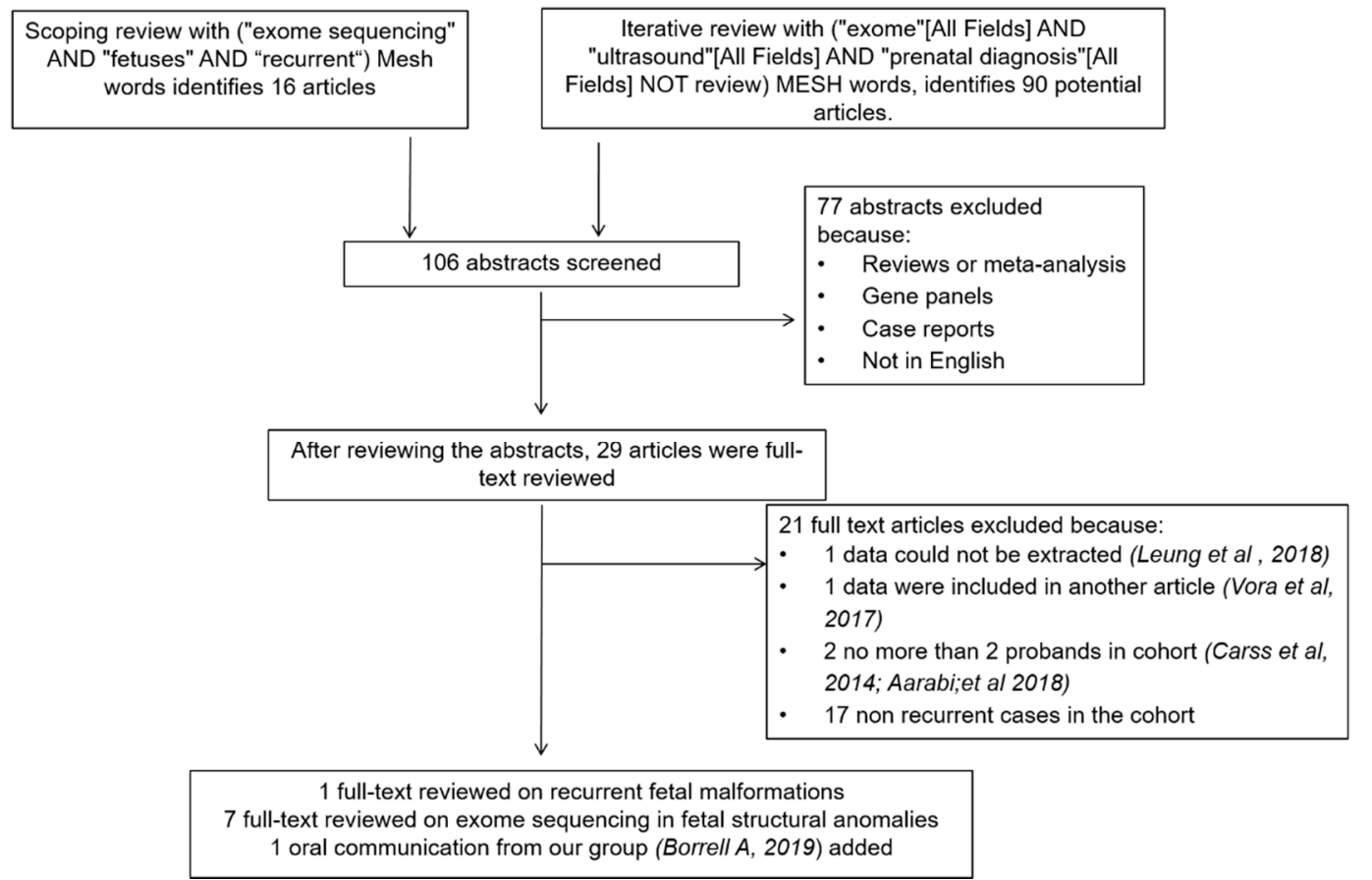

Figure 1. Flowchart summarizing inclusion in this systematic review of studies reporting on diagnostic yield of exome sequencing in fetuses with recurrent structural anomalies and a negative chromosomal microarray analysis or karyotyping, and no family history.

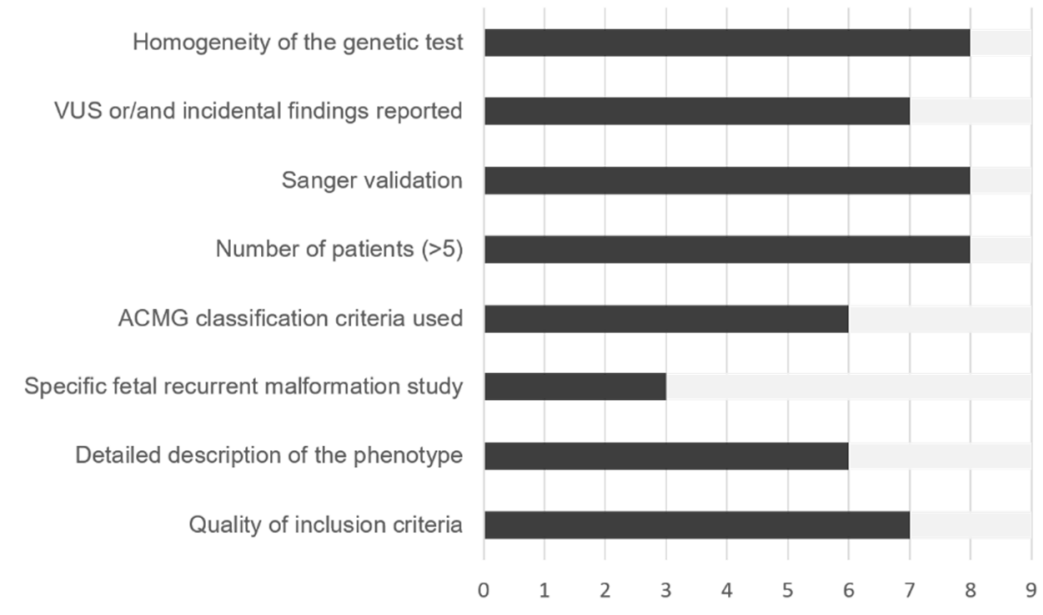

Figure 2. Quality assessment of the nine studies included in this systematic review using eight criteria. VUS: A variant of uncertain (or unknown) significance; ACMG: American College of Medical Genetics and Genomics. 
Table 1. Features of the nine studies included in this systematic review and meta-analysis.

\begin{tabular}{|c|c|c|c|c|c|c|}
\hline Authors & Year & Site & $\begin{array}{c}\text { Fetuses with } \\
\text { Recurrent } \\
\text { Anomalies } \\
(N)\end{array}$ & $\begin{array}{c}\text { Specific } \\
\text { Recurrent } \\
\text { Anomalies } \\
\text { Included }\end{array}$ & $\begin{array}{c}\text { Clinical ES } \\
\text { (CES) or Whole } \\
\text { ES (WES) }\end{array}$ & $\begin{array}{c}\text { Sanger } \\
\text { Validation }\end{array}$ \\
\hline Alamillo et al. [19] & 2015 & $\begin{array}{c}\text { Aliso Viejo, CA, } \\
\text { USA }\end{array}$ & 6 & No & WES-trio & Yes \\
\hline Yates et al. [20] & 2016 & $\begin{array}{l}\text { Gaithersburg, } \\
\text { MD, USA }\end{array}$ & 24 & No & CES & Yes \\
\hline Pangalos et al. [21] & 2016 & Athens, Greece & 3 & No & CES & Yes \\
\hline Meier et al. [22] & 2018 & $\begin{array}{c}\text { Basel, } \\
\text { Switzerland }\end{array}$ & 8 & No & WES-trio & Yes \\
\hline Borrell et al. [28] & 2019 & $\begin{array}{l}\text { Barcelona, } \\
\text { Catalonia, } \\
\text { Spain }\end{array}$ & 12 & Yes & CES-solo & Yes \\
\hline $\begin{array}{c}\text { Corsten-Janssen } \\
\text { et al. [23] }\end{array}$ & 2019 & $\begin{array}{l}\text { Groningen, } \\
\text { Netherlands }\end{array}$ & 4 & No & CES-trio & No \\
\hline Guo et al. [16] & 2019 & Beijing, China & 40 & Yes & WES-trio & Yes \\
\hline Greenbaum et al. [24] & 2019 & $\begin{array}{c}\text { Tel Hashomer, } \\
\text { Israel }\end{array}$ & 15 & No & $\begin{array}{l}\text { WES-trio, } \\
\text { quatro \& solo }\end{array}$ & No \\
\hline Vora et al. [25] & 2020 & $\begin{array}{l}\text { Chapel Hill, } \\
\text { NC, USA }\end{array}$ & 28 & No & CES-trio & Yes \\
\hline
\end{tabular}

CES: clinical exome sequencing; WES: whole-exome sequencing

\subsection{Risk of Bias of Included Studies}

The quality of the studies was assessed using a modified Standards for Reporting of Diagnostic Accuracy (STARD) for this project. We considered the following quality criteria: (a) well defined inclusion criteria; (b) phenotype correctly described; (c) study focused on fetuses with recurrent structural anomalies; (d) genetic variants classified according the ACMG; (e) more than five fetuses included; (f) subsequent Sanger validation; (g) VUS and incidental findings reported; and (h) same previous genetic test applied to all fetuses.

\subsection{Diagnostic Yield in Recurrent Malformation}

Among the nine studies included, the risk of variants likely to be pathogenic or being pathogenic among fetuses with recurrent structural anomalies and normal results at microarray or karyotyping, by the random effects model, was $40 \%$ (95\%CI: $26 \%$ to $54 \%$ ) (Figure 3). The diagnostic yield observed in each of the included studies ranged from $12 \%$ to $67 \%$, being $60 \%$ in the larger study with 40 cases. Heterogeneity was due to sample error rather than a true-effect, and according to the $\mathrm{I}^{2}$, the proportion of heterogeneity was $62 \%$. Publication bias by the linear regression asymmetry test showed no significant quantification of bias (bias: 1.529; $p=0.059$ ), as empirically depicted by the funnel plot (Figure S1).

When the 57 positive cases were grouped according the anatomic systems that were involved in the recurrent structural anomalies, the most commonly observed pattern was multisystem anomalies $(n=31)$, followed by central nervous system $(n=10)$, hydrops $(n=7)$, musculoskeletal $(n=7)$, and cardiac anomalies $(n=2)$. 
Study

Alamillo et al., 2015

Yates et al., 2016

Pangalos et al., 2016

Meier et al., 2018

Borrell et al.. 2019

Corsten-Janssen et al., 2019

Guo et al., 2019

Greenbaum et al., 2019

Vora et al., 2020

\section{Random effects model}

Heterogeneity: $I^{2}=62 \%, \tau^{2}=0.0246, p<0.01$
Cases Reccurent

Risk $\quad 95 \%-C l$ Weight

$0.50[0.12 ; 0.88] \quad 7.9 \%$

$0.12[0.03 ; 0.32] \quad 15.0 \%$

$0.67[0.09 ; 0.99] \quad 4.9 \%$

$0.38[0.09 ; 0.76] \quad 9.4 \%$

$0.25[0.05 ; 0.57] 11.5 \%$

$0.50[0.07 ; 0.93] \quad 6.0 \%$

$0.60[0.43 ; 0.75] \quad 17.0 \%$

$0.53[0.27 ; 0.79] \quad 12.7 \%$

$0.32[0.16 ; 0.52] 15.6 \%$

$0.40[0.26 ; 0.54] 100.0 \%$

\section{$\begin{array}{llll}0.2 & 0.4 & 0.6 & 0.8\end{array}$ \\ Risk increase}

Figure 3. Forest plot of the diagnostic yield of exome sequencing in 140 fetuses with recurrent structural anomalies from nine studies.

\subsection{Monogenic Variants}

Overall, 87 causative variants of the phenotype were described in 57 of the 140 fetuses with recurrent anomalies. In 45 cases (79\%), causative variants were found in genes with an autosomal recessive inheritance pattern; in six in recessive X-linked genes and inherited from a carrier mother (two cases of L1CAM, FOXP3 and one case of OFD1, AMER1); two in dominant X-linked genes (NONO, ZRSR2); and finally, in four cases in dominant inheritance genes (three "de novo" and one maternal germinal mosaicism (TP63)). Among the 45 cases described with autosomal recessive inheritance, homozygosity was identified in $13(29 \%)$ cases, although you must take into account that the largest study, including half of the identified variants, only enrolled non-consanguineous couples [16] (Table 2).

\subsection{Associated Genes and Fetal Structural Anomalies}

The syndrome most frequently found was Meckel syndrome, diagnosed in five fetuses although caused by four different genes (CEP290 in two cases and CC2D2A, TCTN2, and MSK1 in one case each) (Table 2). Four genes were found to be involved in four cases each: the RAPSN gene related to fetal akinesia syndrome II [16,19], the SLC26A3 gene related to congenital chloride diarrhea [16], the L1CAM gene related to hydrocephalus with X-linked inherence $[16,20]$, and the FOXC2 gene related to lymphedema-distichiasis syndrome [25]. Among the 57 fetuses with a positive diagnosis, in $26(46 \%)$ there was a single anatomical system involved, while in the remaining 31 there was more than one. The anatomical systems more frequently involved in structural anomalies were the following: central nervous system $(n=24)$, musculoskeletal (including polydactyly and arthrogryposis) $(n=20)$, hydrops $(n=14)$, nephrourological $(n=13)$, and cardiovascular $(n=10)$. 
Table 2. Eighty-seven causative variants found in the 57 fetuses with recurrent structural anomalies and a positive ES result included in this systematic review.

\begin{tabular}{|c|c|c|c|c|c|c|c|c|}
\hline Author & Phenotype & Gene & Variant & Type of Variant & $\begin{array}{l}\text { Classification of } \\
\text { Variant }\end{array}$ & Inheritance & Zygosity & Syndrome or Disease \\
\hline \multicolumn{9}{|c|}{ Multisystem Multiple Anomalies } \\
\hline Alamillo et al. [19] & Omphalocele, cleft lip and palate & OFD1 & c. $929 \mathrm{~T}>\mathrm{C}$ & Missense & L.Pat. & XLR & Hemizygous & $\begin{array}{l}\text { Oral-facial-digital } \\
\text { syndrome } 1\end{array}$ \\
\hline Alamillo et al. [19] & $\begin{array}{l}\text { Edema, small and bell-shaped chest, } \\
\text { and scalloping of the ribs }\end{array}$ & RAPSN & c. $484 \mathrm{G}>\mathrm{A}$ & Missense & Pat. & AR & Heterozygous & $\begin{array}{l}\text { RAPSN-associated feta } \\
\text { akinesia deformation } \\
\text { sequence }\end{array}$ \\
\hline Yates et al. [20] & $\begin{array}{l}\text { Hydrops, contractures, and echogenic } \\
\text { kidney }\end{array}$ & FOXP3 & c.1009C>T (p.R337X) & Nonsense & Pat & XLR & Hemizygous & IPEX syndrome \\
\hline Yates et al. [20] & $\begin{array}{l}\text { Macrocephaly, hydrocephalus, cleft } \\
\text { lip and palate, cardiac defect, and } \\
\text { bifid thumb }\end{array}$ & AMER1 & c.705delT & Frameshift & Pat & XLR & Hemizygous & $\begin{array}{l}\text { Osteopathia striata } \\
\text { with cranial sclerosis }\end{array}$ \\
\hline Meier et al. [22] & $\begin{array}{l}\text { 1st: Cerebral hypoplasia, cerebellar } \\
\text { hypoplasia, agenesis of occipital } \\
\text { lobes, bilateral renal agenesis, } \\
\text { ureteral agenesis, and uterine } \\
\text { hypoplasia. 2nd: Corpus callosum } \\
\text { agenesis, cerebral hypoplasia, } \\
\text { arhinencephaly, bilateral renal } \\
\text { hypoplasia and cystic dysplasia, } \\
\text { ureteral hypoplasia, uterine } \\
\text { hypoplasia, and vaginal atresia. }\end{array}$ & KIF14 & $\begin{array}{l}\text { NM014875.2: } \\
\text { c.1750_1751delGA;1780A>T }\end{array}$ & Frameshift; missense & Pat.; Pat. & $\mathrm{AR}$ & $\begin{array}{l}\text { Compound } \\
\text { Heterozygous }\end{array}$ & Isolated microcephaly \\
\hline Guo et al. [16] & Hydrocephalus, hydrops & L1CAM & NM_000425.5: c:3581C>T & Frameshift & L.Pat. & XLR & Hemizygous & $\begin{array}{l}\text { Hydrocephalus due to } \\
\text { aqueductal stenosis }\end{array}$ \\
\hline Guo et al. [16] & $\begin{array}{l}\text { Encephalocele and polycystic kidney } \\
\text { dysplasia }\end{array}$ & $C C 2 D 2 A$ & $\begin{array}{c}\text { NM_001080522: } \\
\text { c.1751G>A; c.3293T>G }\end{array}$ & nonsense & Pat.; Pat. & AR & $\begin{array}{l}\text { Compound } \\
\text { Heterozygous }\end{array}$ & Meckel syndrome 6 \\
\hline Guo et al. [16] & $\begin{array}{l}\text { Anencephaly, heart defect, and } \\
\text { polyhydramnios }\end{array}$ & PUS3 & $\begin{array}{l}\text { NM_031307.4: c.838C }>\mathrm{T} ; \\
\text { c.340T>C }\end{array}$ & Nonsense; missense & L.Pat.; L.Pat. & AR & $\begin{array}{l}\text { Compound } \\
\text { Heterozygous }\end{array}$ & $\begin{array}{l}\text { Mental retardation } \\
\text { autosomal recessive } 55\end{array}$ \\
\hline Guo et al. [16] & $\begin{array}{l}\text { Hydrops, intestinal obstruction, and } \\
\text { polyhydramnios }\end{array}$ & SLC26A3 & $\begin{array}{l}\text { NM_000111.3: } \\
\text { 269_270dup; c.1000G }>\mathrm{T}\end{array}$ & Frameshift; nonsense & Pat.; Pat. & AR & $\begin{array}{l}\text { Compound } \\
\text { Heterozygous }\end{array}$ & $\begin{array}{l}\text { Congenital chloride } \\
\text { diarrhea }\end{array}$ \\
\hline Guo et al. [16] & $\begin{array}{l}\text { Encephalocele, hydrocephalus, and } \\
\text { polycystic kidney dysplasia }\end{array}$ & CEP290 & $\begin{array}{l}\mathrm{NM}_{-}^{-} 025114.3: \text { c. } 613 \mathrm{C}>\mathrm{T} ; \\
\text { c.5329C }>\mathrm{T}\end{array}$ & Nonsense; nonsense & Pat.; Pat. & AR & $\begin{array}{l}\text { Compound } \\
\text { Heterozygous }\end{array}$ & Meckel syndrome 4 \\
\hline Guo et al. [16] & $\begin{array}{l}\text { Hydrocephalus, arthrogryposis } \\
\text { multiplex, and talipes }\end{array}$ & KIAA1109 & $\begin{array}{l}\text { NM_015312.3: c.692del; } \\
\text { c.3323+1G>A }\end{array}$ & Frameshift; splicing & L.Pat; L.Pat. & $\mathrm{AR}$ & $\begin{array}{l}\text { Compound } \\
\text { Heterozygous }\end{array}$ & $\begin{array}{l}\text { Alkuraya-Kucinskas } \\
\text { syndrome }\end{array}$ \\
\hline Guo et al. [16] & $\begin{array}{l}\text { Renal agenesis, hemivertebrae, and } \\
\text { right aortic arch }\end{array}$ & KIAA1109 & $\begin{array}{l}\text { NM_015312.3: c.9153del; } \\
\text { c.13849+11G }>C\end{array}$ & Frameshift; splicing & Pat.; VUS & AR & $\begin{array}{l}\text { Compound } \\
\text { Heterozygous }\end{array}$ & $\begin{array}{l}\text { Alkuraya-Kucinskas } \\
\text { syndrome }\end{array}$ \\
\hline Guo et al. [16] & $\begin{array}{l}\text { Deformed rib cage, short ribs, short } \\
\text { long bones, cardiac defect, and } \\
\text { abnormal lung }\end{array}$ & DYNC2H1 & $\begin{array}{l}\text { NM_001080463.2: } \\
\text { c.9929T>C; c.5920G >T }\end{array}$ & Missense; nonsense & VUS; L.Pat. & AR & $\begin{array}{l}\text { Compound } \\
\text { Heterozygous }\end{array}$ & $\begin{array}{l}\text { Short-rib thoracic } \\
\text { dysplasia } 3 \text { with or } \\
\text { without polydactyly }\end{array}$ \\
\hline Guo et al. [16] & $\begin{array}{l}\text { Aplasia/ hypoplasia of the fibula, } \\
\text { ankle contracture }\end{array}$ & $C 2 C D 3$ & $\begin{array}{l}\text { NM_015531.6: c.3741G }>\text { C ; } \\
\text { c.159_160insC }\end{array}$ & Missense; frameshift & L.Pat.; L.Pat. & AR & $\begin{array}{l}\text { Compound } \\
\text { Heterozygous }\end{array}$ & $\begin{array}{l}\text { Orofaciodigital } \\
\text { syndrome XIV }\end{array}$ \\
\hline Guo et al. [16] & $\begin{array}{l}\text { Cardiac defect, short long bones, and } \\
\text { cystic hygroma }\end{array}$ & CO11A2 & $\begin{array}{c}\text { NM_080679.2 } \\
\text { c.1773+8T>A;c.971dup }\end{array}$ & Splicing; frameshift & VUS; L.Pat. & AR & $\begin{array}{l}\text { Compound } \\
\text { Heterozygous }\end{array}$ & Fibrochondrogenesis \\
\hline
\end{tabular}


Table 2. Cont.

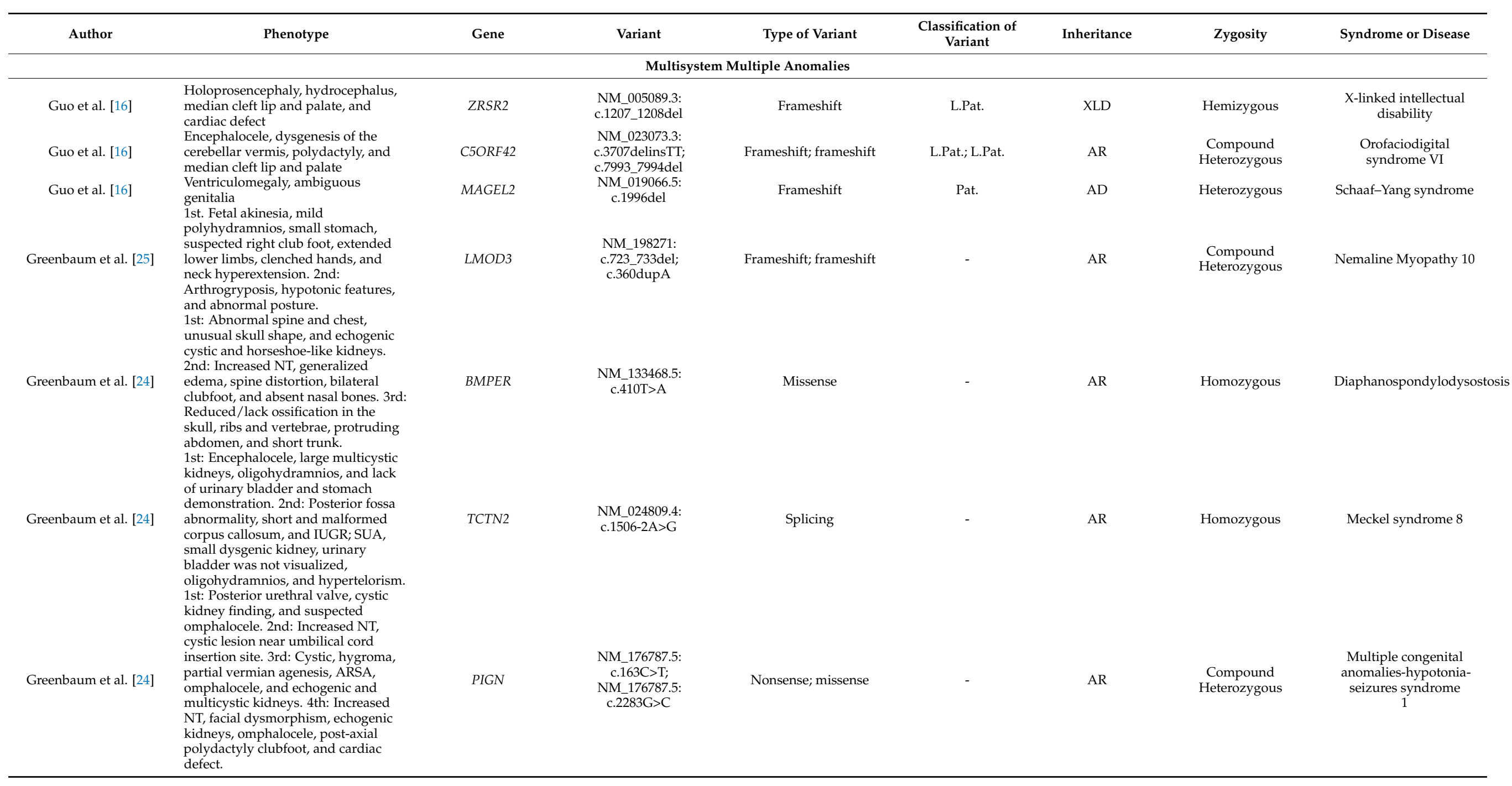


Table 2. Cont.

\begin{tabular}{|c|c|c|c|c|c|c|c|c|}
\hline Author & Phenotype & Gene & Variant & Type of Variant & $\begin{array}{l}\text { Classification of } \\
\text { Variant }\end{array}$ & Inheritance & Zygosity & Syndrome or Disease \\
\hline \multicolumn{9}{|c|}{ Multisystem Multiple Anomalies } \\
\hline Greenbaum et al. [24] & $\begin{array}{l}\text { 1st: Large polycystic kidney, } \\
\text { oligohydramnios, and moderate } \\
\text { bilateral ventriculomegaly. 2nd: } \\
\text { Polycystic kidneys, hydrocephalus, } \\
\text { megacisterna magna, and } \\
\text { macrocephaly. 3rd: Enlarged } \\
\text { echogenic kidneys, severe } \\
\text { oligohydramnios hydrocephalus, } \\
\text { megacisterna magna, and thin corpus } \\
\text { callosum }\end{array}$ & CPT2 & $\begin{array}{l}\text { NM_001330589.1: } \\
\text { c.1239_1240del }\end{array}$ & Frameshift & - & $\mathrm{AR}$ & Homozygous & $\begin{array}{l}\text { CPT II deficiency, lethal } \\
\text { neonatal }\end{array}$ \\
\hline Vora et al. [25] & $\begin{array}{l}\text { Ventriculomegaly, cystic kidneys, } \\
\text { anhydramnios, cardiac defect, and } \\
\text { bilateral polydactyly }(n=4)\end{array}$ & CEP290 & $\begin{array}{l}\text { c.384_387 delTAGA; } \\
\text { (p.Asp128Glufs); c.1936 } \\
\text { C>T (p.Gln646Ter) }\end{array}$ & Frameshift; missense & Pat.; Pat. & $\mathrm{AR}$ & $\begin{array}{l}\text { Compound } \\
\text { Heterozygous }\end{array}$ & Meckel syndrome 4 \\
\hline Vora et al. [25] & $\begin{array}{l}\text { Hand and foot clefting, syndactyly, } \\
\text { facial clefting, and renal pyelectasis ( } n \\
=3 \text { ) }\end{array}$ & TP63 & $\begin{array}{l}\text { c.1028G }>C \\
\text { (p.Arg343Pro) }\end{array}$ & Missense & Pat. & $\mathrm{AD}$ & Heterozygous & $\begin{array}{l}\text { Ectrodactyly, } \\
\text { ectodermal dysplasia, } \\
\text { and cleft/lip }\end{array}$ \\
\hline Vora et al. [25] & Renal agenesis and heart defect & GREPB1L & $\begin{array}{l}\text { c.4881_4882delCA } \\
\text { (p.H1627f); c.277G >A } \\
\text { (p.E92K) }\end{array}$ & Frameshift: missense & L.Pat.; VUS & $\mathrm{AR}$ & $\begin{array}{l}\text { Compound } \\
\text { Heterozygous }\end{array}$ & $\begin{array}{c}\text { Renal } \\
\text { hypoplasia/aplasia }\end{array}$ \\
\hline Vora et al. [25] & $\begin{array}{l}\text { Cystic hygroma, hydrops, complex } \\
\text { heart defect }\end{array}$ & FOXC2 & c.612delC (p.Pro204fs) & & L.Pat. & $\mathrm{AD}$ & Heterozygous & $\begin{array}{l}\text { Lymphedema- } \\
\text { distichiasis } \\
\text { syndrome }\end{array}$ \\
\hline Vora et al. [25] & $\begin{array}{l}\text { Agenesis corpus callosum, shortened } \\
\text { long bones, arthrogryposis, suspected } \\
\text { tetralogy of Fallot, micrognathia, } \\
\text { hypertelorism, kyphoscoliosis, } \\
\text { ambiguous genitalia, and rocker } \\
\text { bottom feet }(n=2)\end{array}$ & ALG3 & $\begin{array}{l}\text { c.518C > T (p.R163C); } \\
\text { c.1185G>C (p.R385T) }\end{array}$ & Missense & VUS; VUS & $\mathrm{AR}$ & $\begin{array}{l}\text { Compound } \\
\text { Heterozygous }\end{array}$ & $\begin{array}{l}\text { Congenital disorder of } \\
\text { glycosylation, 1D }\end{array}$ \\
\hline Vora et al. [25] & $\begin{array}{l}\text { Enlarged bladder with distorted } \\
\text { abdomen, extremely short long bones } \\
\text { and small chest, bilateral polydactyly } \\
\text { on hands, and neck fixed in a flexed } \\
\text { position. Previous pregnancies have } \\
\text { also shown thick NT/ cystic hygroma, } \\
\text { and enlarged cisterna magna with } \\
\text { possible ventriculomegaly. }\end{array}$ & TRAF3iP1 & $\begin{array}{l}\text { c.169G }>\text { A (p.Glu57Lys); } \\
\text { c. } 988-1 \mathrm{G}>\mathrm{C}\end{array}$ & Splicing & VUS: L.Pat. & $\mathrm{AR}$ & $\begin{array}{l}\text { Compound } \\
\text { Heterozygous }\end{array}$ & $\begin{array}{c}\text { Gene typically Senior } \\
\text { Loken syndrome } 9 \text { but } \\
\text { this is a more severe } \\
\text { presentation. } \\
\text { Ciliopathy. }\end{array}$ \\
\hline
\end{tabular}


Table 2. Cont.

\begin{tabular}{|c|c|c|c|c|c|c|c|c|}
\hline Author & Phenotype & Gene & Variant & Type of Variant & $\begin{array}{c}\text { Classification of } \\
\text { Variant }\end{array}$ & Inheritance & Zygosity & Syndrome or Disease \\
\hline \multicolumn{9}{|c|}{ Central Nervous System Anomalies } \\
\hline Yates et al. [20] & Hydrocephalus (aqueductal stenosis) & $L 1 C A M$ & c.2087delG & Frameshift & Pat & XLR & Hemizygous & Hydrocephalus \\
\hline Pangalos et al. [21] & Hydrocephalus +FGR & NEB & $\begin{array}{l}\text { NM_004543.5: } \\
\text { c.11060C>T; } \\
\text { c. } 11333 T>C\end{array}$ & $\begin{array}{l}\text { Canonical missense; } \\
\text { missense }\end{array}$ & L.Pat.; L.Pat. & AR & $\begin{array}{l}\text { Compound } \\
\text { Heterozygous }\end{array}$ & $\begin{array}{l}\text { Nemaline myopathy } \\
\text { (OMIM 2560) (AR) }\end{array}$ \\
\hline Pangalos et al. [21] & Brain MRI abnormalities & ASS1 & $\begin{array}{c}\text { NM_000050: c.725C }>\mathrm{T} \\
\text { c. } 971 \mathrm{G}>\mathrm{T}\end{array}$ & Missense & L.Pat.; Pat. & AR & $\begin{array}{l}\text { Compound } \\
\text { Heterozygous }\end{array}$ & $\begin{array}{l}\text { Citrullinemia (OMIM } \\
\text { 215700) (AR) }\end{array}$ \\
\hline Meier et al. [22] & Meckel-Gruber syndrome like & MSK1 & NM_017777.3:c.417G>A & Splicing & L.Pat. & AR & Homozygous & $\begin{array}{l}\text { Meckel-Gruber } \\
\text { syndrome }\end{array}$ \\
\hline Meier et al. [22] & Dandy-Walker malformation & PIGW & NM178517: c.106A>G & Missense & L.Pat. & AR & Homozygous & $\begin{array}{l}\text { Glycosylphosphatidylinosit } \\
\text { biosynthesis defect } 11\end{array}$ \\
\hline $\begin{array}{l}\text { Corsten-Janssen et al. } \\
{[23]}\end{array}$ & $\begin{array}{l}\text { Cerebellar vermis hypoplasia, } \\
\text { hydronephrosis }\end{array}$ & PEX1 & NM_000466.2:c.2097dupT & Frameshift & - & AR & Homozygous & Zellweger syndrome \\
\hline Guo et al. [16] & Dysgenesis of the cerebellar vermis & POMT1 & $\begin{array}{l}\text { NM_007171.3: } \\
\text { c.110_113dup; } \\
\text { c.169C >T }\end{array}$ & Frameshift; nonsense & Pat.; Pat. & AR & $\begin{array}{l}\text { Compound } \\
\text { Heterozygous }\end{array}$ & $\begin{array}{c}\text { Muscular dystrophy } \\
\text { dystroglycanopathy } \\
\text { type A }\end{array}$ \\
\hline Greenbaum et al. [24] & $\begin{array}{l}\text { Occipital encephalocele, } \\
\text { ventriculomegaly }\end{array}$ & B3GALNT2 & $\begin{array}{l}\text { NM_001277155.2: } \\
\text { c.236-1G>C }\end{array}$ & Splicing & - & AR & Homozygous & $\begin{array}{l}\text { Muscular dystrophy- } \\
\text { dystroglycanopathy }\end{array}$ \\
\hline $\begin{array}{l}\text { Corsten-Janssen et al. } \\
{[23]}\end{array}$ & Severe hydrocephaly & POMGNT1 & $\begin{array}{l}\text { NM_001243766.1: } \\
\text { c.636C >T }\end{array}$ & Synonymous & - & AR & Homozygous & $\begin{array}{l}\text { Walker-Warburg } \\
\text { syndrome }\end{array}$ \\
\hline Borrell et al. [28] & Lissencephaly & $A S P M$ & $\begin{array}{c}\text { NM_018136.4: } \\
\text { c.7551T>G c.9279G }>\text { A }\end{array}$ & Nonsense; nonsense & L.Pat; Pat. & AR & $\begin{array}{l}\text { Compound } \\
\text { Heterozygous }\end{array}$ & $\begin{array}{l}\text { Microcephaly with } \\
\text { simplified gyral pattern }\end{array}$ \\
\hline \multicolumn{9}{|c|}{ Fetal Hydrops } \\
\hline Guo et al. [16] & Hydrops & RAPSN & $\begin{array}{c}\text { NM_032645.5: } \\
\text { c.969C>A; } \\
\text { c.149_153delinsGATGGGC }\end{array}$ & $\begin{array}{l}\text { Nonsense; frameshift } \\
\text { GCTACAAGGAGATGG }\end{array}$ & Pat.; Pat. & AR & $\begin{array}{l}\text { Compound } \\
\text { Heterozygous }\end{array}$ & $\begin{array}{c}\text { Fetal akinesia } \\
\text { deformation sequence } 2\end{array}$ \\
\hline Guo et al. [16] & Hydrops & RYR1 & $\begin{array}{l}\text { NM_001042723.2: } \\
\text { c.2286del; c.6721C>T }\end{array}$ & Frameshift; nonsense & Pat.; Pat. & AR & $\begin{array}{l}\text { Compound } \\
\text { Heterozygous }\end{array}$ & $\begin{array}{l}\text { Multiple pterygium } \\
\text { syndrome lethal type }\end{array}$ \\
\hline Guo et al. [16] & Hydrops fetalis & PIEZO1 & $\begin{array}{l}\text { NM_001142864.4: } \\
\text { c.1536_1537del; } \\
\text { c.4610_4617dup }\end{array}$ & Frameshift; frameshift & L.Pat; L.Pat. & $\mathrm{AD} / \mathrm{AR}$ & $\begin{array}{l}\text { Compound } \\
\text { Heterozygous }\end{array}$ & $\begin{array}{l}\text { Lymphatic } \\
\text { malformation } 6\end{array}$ \\
\hline Vora et al. [25] & Hygroma & FOXC2 & c.251C >T (p.Ala84Val) & & L.Pat. & $\mathrm{AD}$ & Heterozygous & $\begin{array}{l}\text { Lymphedema- } \\
\text { distichiasis } \\
\text { syndrome }\end{array}$ \\
\hline Borrell et al. [28] & Hydrops & SEC23B & $\begin{array}{l}\text { NM_001172745: } \\
\text { c. } 716 \mathrm{~A}>\mathrm{G}\end{array}$ & Missense & L.Pat. & $\mathrm{AR}$ & Homozygous & $\begin{array}{l}\text { Congenital } \\
\text { dyserythropoietic } \\
\text { anemia type II }\end{array}$ \\
\hline Guo et al. [16] & Hydrops & FOXP3 & $\begin{array}{l}\text { NM_014009.4: } \\
\text { c.1120_1122del }\end{array}$ & In frame & L.Pat. & XLR & Hemizygous & $\begin{array}{l}\text { Immunodysregulation, } \\
\text { polyendocrinopathy } \\
\text { and enteropathy, and } \\
\text { X-Linked. }\end{array}$ \\
\hline
\end{tabular}


Table 2. Cont.

\begin{tabular}{|c|c|c|c|c|c|c|c|c|}
\hline Author & Phenotype & Gene & Variant & Type of Variant & $\begin{array}{c}\text { Classification of } \\
\text { Variant }\end{array}$ & Inheritance & Zygosity & Syndrome or Disease \\
\hline \multicolumn{9}{|c|}{ Musculoskeletal Anomalies } \\
\hline Guo et al. [16] & Hemivertebrae & DLL3 & $\begin{array}{l}\text { NM_016941.4: } \\
\text { c.661C }>\mathrm{T}\end{array}$ & Nonsense & Pat. & AR & Homozygous & $\begin{array}{l}\text { Spondylocostal } \\
\text { dysostosis } 1\end{array}$ \\
\hline Guo et al. [16] & Multiple joint contractures & GLDN & $\begin{array}{c}\text { NM_181789.4: } \\
\text { c.1240C>T; c.1027G }>\text { A }\end{array}$ & Nonsense; missense & Pat.; L.Pat. & AR & $\begin{array}{l}\text { Compound } \\
\text { Heterozygous }\end{array}$ & $\begin{array}{l}\text { Lethal Congenital } \\
\text { Contracture } \\
\text { Syndrome } 11\end{array}$ \\
\hline Guo et al. [16] & $\begin{array}{l}\text { Ankle contracture, arthrogryposis } \\
\text { multiplex, scoliosis. }\end{array}$ & CHRNG & $\begin{array}{c}\text { NM_005199.5: c.13C }>\text { T; } \\
\text { c.202C }>\mathrm{T}\end{array}$ & Nonsense; nonsense & L.Pat.; L.Pat. & $\mathrm{AR}$ & $\begin{array}{l}\text { Compound } \\
\text { Heterozygous }\end{array}$ & $\begin{array}{l}\text { Multiple pterygium } \\
\text { syndrome lethal type }\end{array}$ \\
\hline Greenbaum et al. [24] & $\begin{array}{l}\text { 1st: Distal arthrogryposis (hands). } \\
\text { 2nd: Bilateral clubfoot. }\end{array}$ & FKBP14 & $\begin{array}{l}\text { NM_017946.3: } \\
\text { c.568_570del }\end{array}$ & In frame & - & AR & Homozygous & $\begin{array}{l}\text { Ehlers-Danlos } \\
\text { syndrome, } \\
\text { kyphoscoliotic type, } 2\end{array}$ \\
\hline Greenbaum et al. [24] & $\begin{array}{l}\text { 1st: Short long bones, IUFD. 2nd: } \\
\text { Narrow thorax, bowed femur, short } \\
\text { long bones. }\end{array}$ & $E V C 2$ & $\begin{array}{c}\text { NM_147127.4: c.572A; } \\
\text { c.3265C>T }\end{array}$ & Missense; nonsense & - & $\mathrm{AR}$ & $\begin{array}{l}\text { Compound } \\
\text { Heterozygous }\end{array}$ & $\begin{array}{l}\text { Ellis-Van Creveld } \\
\text { syndrome }\end{array}$ \\
\hline Vora et al. [25] & $\begin{array}{l}\text { Arthrogryposis }(n=3) \text {. One with } \\
\text { dextrocardia, partial agenesis of the } \\
\text { right lung. }\end{array}$ & ADGRG6/GPR126 & $\begin{array}{c}\text { c.2515C>T } \\
\text { (p.His839Tyr) }\end{array}$ & Missense & VUS & $\mathrm{AR}$ & Homozygous & $\begin{array}{c}\text { Lethal Congenital } \\
\text { Contracture Syndrome } \\
9\end{array}$ \\
\hline Borrell et al. [28] & Arthrogryposis multiplex & DOK7 & $\begin{array}{l}\text { NM_001164673: } \\
\text { c.230C>T; NM_173660: } \\
\text { c.532+4A>G }\end{array}$ & $\begin{array}{l}\text { Missense; non coding } \\
\text { variant }\end{array}$ & L.Pat.; L.Pat. & $\mathrm{AR}$ & $\begin{array}{l}\text { Compound } \\
\text { Heterozygous }\end{array}$ & $\begin{array}{c}\text { Fetal akinesia } \\
\text { deformation sequence }\end{array}$ \\
\hline \multicolumn{9}{|c|}{ Cardiac Defects } \\
\hline Guo et al. [16] & Cardiac defect & NODAL & $\begin{array}{c}\text { NM_018055: c.823C }>T ; \\
\text { c.172_174del }\end{array}$ & Missense; in frame & Pat.; VUS & $\mathrm{AD} / \mathrm{AR}$ & $\begin{array}{l}\text { Compound } \\
\text { Heterozygous }\end{array}$ & Heterothaxis visceral 5 \\
\hline
\end{tabular}

AR: Autosomal Recessive; AD: Autosomal Dominant; XLD: X-linked Dominant, XLR: X-linked Recessive. 


\section{Discussion}

Our systematic review and meta-analysis of ES/GS in fetuses with a recurrent structural anomaly, a normal result at microarray, and no family disease or Mendelian inheritance identified revealed a $40 \%$ (95\%CI: $26 \%-54 \%$ ) diagnostic yield. In most of the positive cases $(79 \%)$, an autosomal recessive inheritance was identified, although homozygous variants were only found in $29 \%$ of those cases. The high incidence of autosomal recessive diseases was expected since this review includes recurrent fetal malformations from healthy parents. This incidence is expected to depend on the degree of consanguinity and the frequency of autosomal recessive carriers of a given population. Another factor that can explain the wider range of diagnostic yields (12\% to $67 \%)$ observed among the included series is the different type of recurrent structural anomalies included. Multisystem anomalies appear to carry a higher diagnostic yield since they account for more than half of the positive cases.

Meckel syndrome was the most frequently found monogenic disease in this systematic review and accounted for five of the 57 cases reported. Meckel syndrome (OMIM PS249000), also known as Meckel-Gruber syndrome, is a lethal autosomal recessive syndrome that represents the most severe condition in a group of disorders collectively termed the ciliopathies. Meckel syndromre is characterized by the triad of cystic renal disease, posterior fossa abnormalities (usually occipital encephalocele), and hepatic ductal plate malformation, leading to hepatic fibrosis and bile duct proliferation. Polydactyly is relatively common. The prevalence is estimated at $1 / 38,500$ births in Europe [29], although it can be higher in specific populations with a high consanguinity rate such as Gujarati Indians (1/1300) or Qatar (1/5000) [30]. A large European series with 191 Meckel syndrome cases noted that $90 \%$ of them were diagnosed prenatally at a mean gestational age of 14.3 (range 11-36) weeks [29]. Hence, the reason for the high representation of Meckel syndrome in this review may be its characteristic ultrasound pattern, which may facilitate an easy prenatal recognition rather than a high prenatal prevalence [31].

A large prospective study carried out in the Spanish population demonstrated the frequency of carriers of prevalent diseases. The autosomal recessive diseases reported with a carrier frequency higher than 1/40 were the following: GJB2-related DFNB1 nonsyndromic hearing loss and deafness, cystic fibrosis, alpha-thalassemia, phenylketonuria, spinal muscular atrophy, familial Mediterranean fever, and autosomal recessive polycystic kidney disease (ARPKD) [31]. Although prevalent in this population, they have not been described in this review because affected fetuses do not typically present with structural anomalies. Although autosomal dominant polycystic kidney disease can be easily recognized by ultrasound, prenatal diagnosis of ARPKD is currently unreliable. Preconceptional or prenatal screening of carriers of autosomal recessive and X-linked diseases can identify potential affected fetuses irrespective of their ultrasound expressivity. The reason why carrier screening in Europe and other world regions is only offered to couples conceiving by assisted reproduction techniques is unclear.

Currently, ES is being increasingly applied to prenatal diagnosis, as "whole ES" when all the exons are studied, "clinical ES" when only the exons of the OMIM genes are interpreted, or even as "gene panel" when specific genes are selected according to the fetal phenotype. Gene panels were not included in this study, although skeletal, hydrops, or nephrourological panels could be helpful in fetuses with structural anomalies involving a single anatomical system, which in this review account for half of the positive cases. Among the nine studies included, five used clinical ES and four whole ES, and the trio approach was the most used. There is strong evidence of ES being a powerful tool in discovering the underlying etiology of recurrent fetal malformations detected by prenatal ultrasonography. Expansion from ES that only covers mutations in coding regions ( $\sim 97 \%$ of exons) to GS, which also covers noncoding regions of the genome in the near future, is expected to increase the diagnostic yield. It cannot reliably detect copy-number variants at the single gene level. 
A fact that makes the prenatal identification of monogenic syndromes difficult is the discordance between the fetal phenotype and the pediatric or adult phenotype, and this is not well described, yet. The authors of the largest study included in this review, Guo et al., reported four genes (PUS3, SZT2, LAMA5, and ZRSR2) that were found to show discordant phenotypes between the prenatal and postnatal periods, suggesting new relationships between genes and phenotypes in the fetal stage, which may expand the spectrum of the disease [16].

\section{Conclusions}

This systematic review and meta-analysis demonstrate that the ES technique is particularly useful when applied to prenatal diagnosis of monogenic syndromes, due to the lack of well-understood phenotypes in this early stage of human development. ES has been shown to improve the diagnostic yield of recurrent fetal structural anomalies, to be able to discover new genes potentially relevant in human development, and to be able to identify new pathogenic variants.

Supplementary Materials: The following are available online at https:/ / www.mdpi.com/article/ $10.3390 / \mathrm{jcm} 10204739 / \mathrm{s} 1$, Supplementary S1: Search strategy and query syntaxes used in this systematic review, Figure S1: Funnel plot analysis for the asymmetry of publication among small studies.

Author Contributions: Conceptualization: A.B., M.P.; Data curation and Formal analysis: R.J.M.-P. and M.P.; Validation: A.B.; Writing—original draft: all authors. All authors have read and agreed to the published version of the manuscript.

Funding: This study has been funded by Instituto de Salud Carlos III through the project "PI17/01153" (Co-funded by European Regional Development Fund/European Social Fund "A way to make Europe" / Investing in your future").

Institutional Review Board Statement: Not applicable.

Informed Consent Statement: Not applicable.

Data Availability Statement: All data generated are included in this article and its supplementary material files. Further enquiries can be directed to the corresponding author.

Acknowledgments: The authors' thank Jane Juusola, Erin Ryan, and Neeta L Vora and colleagues for share with us their data to allow complete our analysis.

Conflicts of Interest: The authors report no conflict of interest.

\section{References}

1. Dolk, H.; Loane, M.; Garne, E. The prevalence of congenital anomalies in Europe. Adv. Exp. Med. Biol. 2010, 686, 349-364. [CrossRef]

2. Nicolaides, K.H.; Snijders, R.J.M.; Campbell, S.; Gosden, C.M.; Berry, C. Ultrasonographically detectable markers of fetal chromosomal abnormalities. Lancet 1992, 340, 704-707. [CrossRef]

3. Wapner, R.J.; Martin, C.L.; Levy, B.; Ballif, B.C.; Eng, C.M.; Zachary, J.M.; Savage, M.; Platt, L.D.; Saltzman, D.; Grobman, W.A.; et al. Chromosomal microarray versus karyotyping for prenatal diagnosis. N. Engl. J. Med. 2012, 367, 2175-2184. [CrossRef] [PubMed]

4. Callaway, J.L.A.; Shaffer, L.G.; Chitty, L.S.; Rosenfeld, J.A.; Crolla, J.A. The clinical utility of microarray technologies applied to prenatal cytogenetics in the presence of a normal conventional karyotype: A review of the literature. Prenat. Diagn. 2013, 33, 1119-1123. [CrossRef] [PubMed]

5. Glinianaia, S.V.; Tennant, P.W.G.; Rankin, J. Risk estimates of recurrent congenital anomalies in the UK: A population-based register study. BMC Med. 2017, 15, 1-14. [CrossRef] [PubMed]

6. Bossuyt, P.M.; Reitsma, J.B.; Bruns, D.E.; Gatsonis, C.A.; Glasziou, P.P.; Irwig, L.M.; Lijmer, J.G.; Moher, D.; Rennie, D.; De Vet, H.C.W. Towards Complete and Accurate Reporting of Studies of Diagnostic Accuracy: The STARD Initiative. Radiology 2003, 226, 24-28. [CrossRef] [PubMed]

7. Nyaga, V.N.; Arbyn, M.; Aerts, M. Metaprop: A Stata command to perform meta-analysis of binomial data. Arch. Public Health 2014, 72, 1-10. [CrossRef]

8. Newcombe, R.G. Two-sided confidence intervals for the single proportion: Comparison of seven methods. Stat. Med. 1998, 17, 857-872. [CrossRef] 
9. Borenstein, M.; Hedges, L.V.; Higgins, J.P.T.; Rothstein, H.R. Fixed-Effect versus Random-Effects Models. In Introduction to Meta-Analysis; John Wiley \& Sons: Hoboken, NJ, USA, 2009.

10. Stuck, A.E.; Rubenstein, L.Z.; Wieland, D. Bias in meta-analysis detected by a simple, graphical test. Asymmetry detected in funnel plot was probably due to true heterogeneity. BMJ 1998, 316, 469. [CrossRef]

11. Biljana, M.; Jelena, M.; Branislav, J.; Milorad, R. Bias in meta-analysis and funnel plot asymmetry. In Proceedings of the Studies in Health Technology and Informatics, Ljubljana, Slovenia, 1 January 1999.

12. Egger, M.; Smith, G.D.; Schneider, M.; Minder, C. Bias in meta-analysis detected by a simple, graphical test. Br. Med. J. 1997, 315, 629-634. [CrossRef]

13. Copas, J. Meta-analysis, funnel plots and sensitivity analysis. Biostatistics 2000, 1, 247-262. [CrossRef]

14. Carpenter, J.R.; Schwarzer, G.; Rücker, G.; Künstler, R. Empirical evaluation showed that the Copas selection model provided a useful summary in 80\% of meta-analyses. J. Clin. Epidemiol. 2009, 62, 624-631. [CrossRef] [PubMed]

15. Schwarzer, G. Meta: An R package for meta-analysis. R News 2007, 7, 40-45.

16. Guo, W.; Lai, Y.; Yan, Z.; Wang, Y.; Nie, Y.; Guan, S.; Kuo, Y.; Zhang, W.; Zhu, X.; Peng, M.; et al. Trio-whole-exome sequencing and preimplantation genetic diagnosis for unexplained recurrent fetal malformations. Hum. Mutat. 2020, 41, 432-448. [CrossRef] [PubMed]

17. Yang, X.; Huang, L.Y.; Pan, M.; Xu, L.L.; Zhen, L.; Han, J.; Li, D.Z. Exome sequencing improves genetic diagnosis of fetal increased nuchal translucency. Prenat. Diagn. 2020, 40, 1426-1431. [CrossRef] [PubMed]

18. Choy, K.W.; Wang, H.; Shi, M.; Chen, J.; Yang, Z.; Zhang, R.; Yan, H.; Wang, Y.; Chen, S.; Kin Chau, M.H.; et al. Prenatal diagnosis of fetuses with increased nuchal translucency by genome sequencing analysis. Front. Genet. 2019, 10, 761. [CrossRef] [PubMed]

19. Alamillo, C.L.; Powis, Z.; Farwell, K.; Shahmirzadi, L.; Weltmer, E.C.; Turocy, J.; Lowe, T.; Kobelka, C.; Chen, E.; Basel, D.; et al. Exome sequencing positively identified relevant alterations in more than half of cases with an indication of prenatal ultrasound anomalies. Prenat. Diagn. 2015, 35, 1073-1078. [CrossRef]

20. Yates, C.L.; Monaghan, K.G.; Copenheaver, D.; Retterer, K.; Scuffins, J.; Kucera, C.R.; Friedman, B.; Richard, G.; Juusola, J. Whole-exome sequencing on deceased fetuses with ultrasound anomalies: Expanding our knowledge of genetic disease during fetal development. Genet. Med. 2017, 19, 1171-1178. [CrossRef]

21. Pangalos, C.; Hagnefelt, B.; Lilakos, K.; Konialis, C. First applications of a targeted exome sequencing approach in fetuses with ultrasound abnormalities reveals an important fraction of cases with associated gene defects. PeerJ 2016, 4, e1955. [CrossRef]

22. Meier, N.; Bruder, E.; Lapaire, O.; Hoesli, I.; Kang, A.; Hench, J.; Hoeller, S.; De Geyter, J.; Miny, P.; Heinimann, K.; et al. Exome sequencing of fetal anomaly syndromes: Novel phenotype-genotype discoveries. Eur. J. Hum. Genet. 2019, 27, 730-737. [CrossRef]

23. Corsten-Janssen, N.; Bouman, K.; Diphoorn, J.C.D.; Scheper, A.J.; Kinds, R.; el Mecky, J.; Breet, H.; Verheij, J.B.G.M.; Suijkerbuijk, R.; Duin, L.K.; et al. A prospective study on rapid exome sequencing as a diagnostic test for multiple congenital anomalies on fetal ultrasound. Prenat. Diagn. 2020, 40, 1300-1309. [CrossRef]

24. Greenbaum, L.; Pode-Shakked, B.; Eisenberg-Barzilai, S.; Dicastro-Keidar, M.; Bar-Ziv, A.; Goldstein, N.; Reznik-Wolf, H.; Poran, H.; Rigbi, A.; Barel, O.; et al. Evaluation of Diagnostic Yield in Fetal Whole-Exome Sequencing: A Report on 45 Consecutive Families. Front. Genet. 2019, 10, 425. [CrossRef]

25. Vora, N.L.; Gilmore, K.; Brandt, A.; Gustafson, C.; Strande, N.; Ramkissoon, L.; Hardisty, E.; Foreman, A.K.M.; Wilhelmsen, K.; Owen, P.; et al. An approach to integrating exome sequencing for fetal structural anomalies into clinical practice. Genet. Med. 2020, 22, 954-961. [CrossRef]

26. Hillman, S.C.; Willams, D.; Carss, K.J.; Mcmullan, D.J.; Hurles, M.E.; Kilby, M.D. Prenatal exome sequencing for fetuses with structural abnormalities: The next step. Ultrasound Obstet. Gynecol. 2015, 45, 4-9. [CrossRef]

27. Aarabi, M.; Sniezek, O.; Jiang, H.; Saller, D.N.; Bellissimo, D.; Yatsenko, S.A.; Rajkovic, A. Importance of complete phenotyping in prenatal whole exome sequencing. Hum. Genet. 2018, 137, 175-181. [CrossRef] [PubMed]

28. Borrell, A.; Pauta, M.; Nadal, A.; Arca, G.; Paz, F.; Miño, Y.; Segura, M.; Marimón, E.; Pina, S.; Tubau, A.; et al. OC06.01: Single gene, gene panel and exome sequencing applied in structurally abnormal fetuses with a normal chromosomal microarray analysis. Ultrasound Obstet. Gynecol. 2019, 54, 13-14. [CrossRef]

29. Barisic, I.; Boban, L.; Loane, M.; Garne, E.; Wellesley, D.; Calzolari, E.; Dolk, H.; Addor, M.C.; Bergman, J.E.H.; Braz, P.; et al. Meckel-Gruber Syndrome: A population-based study on prevalence, prenatal diagnosis, clinical features, and survival in Europe. Eur. J. Hum. Genet. 2015, 23, 746-752. [CrossRef] [PubMed]

30. Al-Belushi, M.; Al Ibrahim, A.; Ahmed, M.; Ahmed, B.; Khenyab, N.; Konje, J.C. A review of Meckel-Gruber syndrome-Incidence and outcome in the state of Qatar. J. Matern.-Fetal Neonatal Med. 2016, 29, 2013-2016. [CrossRef]

31. Hansen, C.C.; Søreide, K. Systematic review of epidemiology, presentation, and management of Meckel's diverticulum in the 21st century. Medicine 2018, 97, e12154. [CrossRef] [PubMed] 